\title{
Direct identification of interfacial degradation in blue phosphorescent OLEDs using nanoscale chemical depth profiling
}

\section{Soohwan Sul}

Samsung Advanced Institute of Technology, SEC https://orcid.org/0000-0001-7466-9151

\section{Gustavo Trindade}

National Physical Laboratory https://orcid.org/0000-0001-6998-814X

Joonghyuk Kim

Samsung Electronics Co., LTD

\section{Rasmus Havelund}

National Physical Laboratory

\section{Sungjun Park}

Samsung Advanced Institute of Technology, SEC

\section{Youngsik Shin}

Samsung Advaned Institute of Technology

\section{Hyejin Bae}

Samsung Advanced Institute of Technology

\section{Young Mo Sung}

Samsung Advanced Institute of Technology, SEC https://orcid.org/0000-0002-8362-6687

\section{Lidija Matjacic}

National Physical Laboratory

\section{Yongsik Jung}

Samsung Advanced Institute of Technology https://orcid.org/0000-0003-4496-2351

\section{Jung Yeon Won}

Samsung Advanced Institute of Technology

\section{Woo Sung Jeon}

Samsung Advanced Institute of Technology

\section{Hyeonho Choi}

Samsung Electronics Co., Ltd. https://orcid.org/0000-0002-6884-8193

\section{Hyo Sug Lee}

Samsung Advanced Institute of Technology

Jae-Cheol Lee

Samsung Advanced Institute of Technology

Jung-Hwa Kim 
Samsung Advanced Institute of Technology https://orcid.org/0000-0002-2402-9508

lan Gilmore ( $\square$ ian.gilmore@npl.co.uk)

National Physical Laboratory https://orcid.org/0000-0002-0981-2318

\section{Article}

Keywords:

Posted Date: February 2nd, 2022

DOl: https://doi.org/10.21203/rs.3.rs-1279729/v1

License: (c) (1) This work is licensed under a Creative Commons Attribution 4.0 International License. Read Full License 


\section{Abstract}

Understanding the degradation mechanism of organic light-emitting diodes (OLED) is essential to improve device performance and stability. OLED failure, if not process-related, arises mostly from chemical instability. However, the challenges of sampling from nanoscale organic layers and interfaces with enough analytical information has hampered identification of degradation products and mechanisms. Here, we present a high-resolution diagnostic method of OLED degradation using an Orbitrap mass spectrometer equipped with a gas cluster ion beam to gently desorb nanometre levels of materials, providing unambiguous molecular information with 7-nm depth resolution. We measured blue phosphorescent OLED devices and showed that dominant chemical degradation occurred at the interface between electron transport and emission layers $(E M L / E T L)$ where exciton distribution was maximised. We also show an approximately two orders of magnitude increase in lifetime of a device with slightly modified host material, which presented negligible EML/ETL interfacial degradation. Our results provide insight for material and device architecture development.

\section{Introduction}

Organic light emitting diodes (OLEDs) have become widely used in the display and lighting industry. Their performance, energy-efficiency, and manufacturing scalability have enabled commercial success in mobile phones and tablet displays, but the challenge of insufficient device lifetimes still remains for many future applications requiring higher luminance or higher emission energy such as outdoor lighting/displays and blue OLEDs. In particular, blue OLEDs with phosphorescent or thermally-assisted delayed fluorescent (TADF) emitters that are highly energy efficient compared to commercially available fluorescence-type blue OLEDs have suffered from decaying luminance which leads to fading and, eventually, failing devices. ${ }^{1}$ Significant effort has been dedicated to reducing this degradation to extend the operational device lifetime comparable to phosphorescent red and green OLEDs. ${ }^{2-5}$

In modern OLED devices, the extrinsic degradation mechanisms mainly relate to device processing, such as ingress of water and oxygen, impurities, and poor encapsulation, are well understood and now controlled to the extent that the main mechanisms contributing to the degradation are intrinsic to the device. These include photo- and electro-chemical degradation due to high exciton density, high energy localized excitons, or high energy polarons, electric-field induced inter-layer diffusion of mobile ions, and heat-induced morphological change during device operation. ${ }^{1,6-8}$ Much has been learned about the intrinsic mechanisms by varying device structure and material selection. Aziz et al. ${ }^{9}$ showed that the transport of holes into a layer of the electroluminescent molecule $\mathrm{Alq}_{3}$ causes the formation of degradation products that quench fluorescence. This explained the improved lifetime for a device with an emissive layer consisting of a hole-transporting material (host) mixed with the $\mathrm{Alq}_{3}$ (dopant) over a device with separate layers of the same materials. In a recent study of blue OLEDs with mixed host:dopant emissive layers, Kim et al. ${ }^{10}$ showed that degradation may occur through reductive quenching of a host exciton to form a radical ion pair (host ${ }^{\star}+$ dopant $_{\rightarrow}$ host $^{\bullet+}+$ dopant $^{\mathbf{\bullet}^{-}}$). Instable 
radicals can return to the uncharged ground state through electron transfer from the dopant to the host but this occurs in competition with irreversible degrading reactions, e.g. bond scission, leading to loss of emitters and potentially the formation of fluorescence, or phosphorescence quenching species. Thanks to these research efforts to understand and to reduce degradation, the device lifetimes of red and green OLEDs approaching hundreds of thousand hours are now attainable, although better device efficiency and lifetime are required for blue OLEDs. ${ }^{1}$ Nevertheless, it still remains as a scientific challenge to understand the origin and underlying mechanism for device degradation in OLEDs.

Mass spectrometry has been widely applied to identify reaction products or changes in chemical compositions to understand the pathways involved in degradation within organic semiconductor devices. Approaches include analysis from a solution of the small molecules using liquid chromatography mass spectrometry (LC-MS) $)^{11-13}$ analysis of processed materials using direct analysis of the device using laser desorption/ionization time-of flight mass spectrometry (LDI-ToF-MS). ${ }^{14-16}$ Those are useful but give little or no information concerning where in the devices the reactions occur. Recent studies by LDI-ToF-MS and desorption electrospray ionization mass spectrometry (DESI-MS) demonstrated 3D profiling of aged OLED devices with limited depth resolution of hundreds of nanometres, providing the lateral and axial distribution of degradation products. ${ }^{17,18}$ In recent years, secondary ion mass spectrometry (SIMS) has become a powerful capability for chemical depth profiling and 3D imaging of organic electronic devices. The method involves using an energetic focused beam of primary ions directed at the surface. This causes local desorption, or sputtering, of so-called secondary ions from the near-surface (several nanometres) that are detected in a mass spectrometer, typically a time-of-flight (ToF) design. A gas cluster ion beam (GCIB) is often used in combination to gently sputter away surface material without causing significant sub-surface damage and permits mass spectra to be obtained from organic electronic devices in a layer-by-layer manner. ${ }^{13,19-22}$ Whilst ToF-SIMS has made important contributions towards resolving organic device degradation problems, ${ }^{23,24}$ it has relatively low mass resolving power $(m / \Delta m \sim$ $10,000)$ and mass accuracy (10-30 ppm), which limits the identification of complex molecules.

We have recently developed a next generation instrument, the OrbiSIMS, ${ }^{25}$ that allows sputtering by the "gentle" argon cluster ion beam with direct analysis using a high-performance Orbitrap ${ }^{\mathrm{TM}} \mathrm{HF}$ mass spectrometer with a mass-resolving power, $m / \Delta m$, of $\sim 240,000$ (at $m / z 200$ ) and a mass accuracy better than $2 \mathrm{ppm} .{ }^{25}$. We aim to achieve spectra with quality comparable to high-performance LC-MS from sub$10 \mathrm{~nm}$ layer of organic material to enable localisation and identification of reaction products by sampling from nanoscale organic layers and their interfaces in degraded OLED. For comparison, LC-MS data is also acquired of the pristine and aged devices.

\section{Results}

Substrates with four devices were fabricated as described in Methods. To follow and quantify the degradation, the electroluminescence (EL) as well as device characteristics were measured, and two devices were driven at a constant current with a luminance of $1000 \mathrm{~cd} \mathrm{~m}^{-2}$ until their $95 \%\left(T_{95}\right)$ and $70 \%$ 
electroluminescence levels $\left(T_{70}\right)$ were reached. One device, $\mathrm{PL}_{55}$, was degraded under irradiation of 400 nm laser until a $55 \%$ photoluminescence level $(\mathrm{PL})$ was reached for comparison. The fourth device, $\mathrm{T}_{100}$, was left pristine. The degradation is summarised in Supplementary Table 1. Performance characteristics of the $T_{100}, T_{95}$, and $T_{70}$ devices including external quantum efficiency (EQE), current efficiency, $E L$ spectrum, and current density-voltage plot are provided in Supplementary Figure 1. The pristine device showed a typical external quantum efficiency of $17.4 \%$ at $1000 \mathrm{~cd} \mathrm{~m}^{-2}$ with a maximum EQE of $19.1 \%$, indicating that the blue OLED device with an Ir complex emitter utilized most of injected electrons and holes for phosphorescent emission and its internal quantum efficiency (IQE) was close to unity. In spite of the decent EQE of the blue OLED, its operational lifetime was limited to 1.5 - 2 hours for $T_{70}$.

To understand the physical and chemical origin of the blue OLED device degradation, we investigated the device characteristics and chemical changes. The max. EQE of the blue OLED decreased gradually from $19.1-12.8 \%$ and the driving voltage at a constant luminescence condition increased from $5.83 \mathrm{~V}$ to $6.10 \mathrm{~V}$ upon the device degradation from $T_{100}$ to $T_{70}$ (See Supplementary Table 1 and Supplementary Figure 1). The EL intensity showed a gradual decrease with device aging, but the normalized EL spectra were identical to each other regardless of the degradation. Although these data provided typical device characteristics and performance evaluation, the underlying origin of the short operational lifetime for the blue device is still elusive, requiring further physical and chemical analysis. High-resolution mass spectra were acquired from a $200 \mu \mathrm{m} \times 200 \mu \mathrm{m}$ area successively deeper into the device using a $5 \mathrm{keV} \mathrm{Ar}_{2500}{ }^{+}$ gas cluster ion beam for sputtering with analysis using the Orbitrap HF mass spectrometer (Figure 1a). Each spectrum consumes a remarkably small amount of material, approximately $0.17 \mathrm{~nm}$ thickness of material (equivalent to $6.8 \mu \mathrm{m}^{3}$ ). A reference device containing a $1 \mathrm{~nm}$-thick blue Ir dopant layer in a host matrix with a total thickness of $50 \mathrm{~nm}$ was used (Figure 1b) to evaluate the depth resolution under these conditions (FWHM $~ 7 \mathrm{~nm}$ ). Depth profiles were obtained from all four devices. A positive ion depth profile (Figure 1c) from $\mathrm{T}_{100}$ pristine device shows that the chemistry of the different layers can be resolved in agreement with the schematic of the device multilayer architecture shown in Figure 1c. The low fragmentation from the argon cluster sputtering and high-resolution Orbitrap MS results in highresolution LC-MS quality spectra with $\sim 7 \mathrm{~nm}$ depth resolution. The organic materials in each layer give rise to molecular or pseudo-molecular ion peaks that are readily identified based on their accurate mass with sub-ppm mass resolution (Supplementary Figures 2-3 and Supplementary Tables 2-3) and example spectra with peaks from all regions in the device are shown (Figure 1d).

To check the technical repeatability, three repeat depth profiles were recorded from the same device (Supplementary Figure 4). The profiles are essentially indistinguishable from each other demonstrating good measurement repeatability. On first inspection, the depth profile from a degraded device has only minor differences to the pristine device $\left(T_{100}\right)$, however, the spectra from the emissive layers differ in detail. To compare the spectra, each spectrum is first normalised to its total intensity to account for any variation related to the measurement, e.g. primary beam current variation. Five peaks at $\mathrm{m} / z 369.1038$, $\mathrm{m} / z$ 577.1679, $\mathrm{m} / z$ 943.2475, $\mathrm{m} / \mathrm{z} 1019.2789$ and $\mathrm{m} / \mathrm{z} 1127.2924$ have significantly higher normalised intensities in the spectra from $\mathrm{T}_{70}$ and $\mathrm{PL}_{55}$ than in the spectrum from the pristine device. Using the 
accurate mass measurement and the known initial composition of the layers where the ions are detected, putative mass and structure assignments are given (Table 1, Figure 2a-b) They are all potential reaction products of DBFPO (derivatives of DBFPO:Liq) which is in the hole blocking and electron transport layers ( $H B L$ and ETL, respectively). DBFPO consisting of diplenylphosphine oxide and dibenzofuran groups served often as ETL and ambipolar host. ${ }^{18,23}$ The normalised intensities for these peaks are, on average, between 14 and 120 times higher in the spectrum from $\mathrm{PL}_{55}$, and between 5 and 23 times higher in the spectrum from $T_{70}$ than the pristine device (Figure $2 \mathrm{c}$ ). The effect of the degradation of $T_{95}$ are not well pronounced. However, it can reasonably be postulated that changes observed at the EL $70 \%$ degradation level predominantly result from mechanisms that are also in play during the initial degradation. Chemical analysis of OLED degradation has in other studies required further device aging, even to the $T_{10}$ level of degradation. ${ }^{13,19,26}$

Depth profiles for each device of the five degradation products (Figure $2 b$ ) reveal a localisation to the ETL/EML (emissive layer) interface, suggesting that the ions may be degradation products from ETL, $H B L$, and EML materials. There are common features as well as some differences in the depth profiles. Regardless of the aging method - current-driven or photo-irradiated, we found that the dominant degradation products were identical. Subtle differences were observed in the depth profiles. For example, the $m / z 369.1038$ is located deeper in the EML than the other degradation products. In $\mathrm{PL}_{55}$, the $m / z$ 577.1679 is observed with much higher intensity and before the EML is reached, compared with the $T_{70}$ device where it is distinctly in the EML. This suggests that the photo-irradiation of $\mathrm{PL}_{55}$ has caused the formation of the degradation product in the electron injection and electron transport layers. This degradation is less intense and is not formed in these layers in electrically-driven devices and it is pronounced when the device was irradiated by a 400-nm laser (bandwidth $\sim 20 \mathrm{~nm}$ ). Considering the wavelength of the laser and energy levels of layer materials ${ }^{13}, \mathrm{HTL}$ (NPB, $\left.3.1 \mathrm{eV}\right)$, blue Ir dopant (2.9 eV), and ETL (DBFPO:Liq, $3.4 \mathrm{eV}$ ) may absorb the intense light, which leads to material degradation through high energy exciton generation via exciton-exciton annihilation or photo-thermal effect. ${ }^{27}$

We also performed unsupervised multivariate analysis ${ }^{28}$ of a combined dataset with depth profiles for each device to identify thermal degradation products in the NPB layer of the $\mathrm{PL}_{55}$ device, which are likely induced by the laser irradiation ${ }^{29}$ (Supplementary note 1 and Supplementary Figures 5-6).

For comparison with previous studies, the devices were also analysed by traditional LC-MS methods for bulk analysis (Supplementary Figure 7). However, the subtle differences discovered in the OrbiSIMS depth resolved data were not observed, in particular, the reaction products from aged devices. 
Table 1

Putative assignments for potential degradation products in the blue OLED device.

\begin{tabular}{|llll|}
\hline $\mathbf{m} / \mathbf{z}$ & Assignment & & Mass deviation (ppm) \\
\hline 369.1038 & $\mathrm{C}_{24} \mathrm{H}_{18} \mathrm{PO}_{2}{ }^{+}$ & {$[\mathrm{M}-\mathrm{R}]^{+}, \mathrm{R}=\mathrm{C}_{12} \mathrm{H}_{8} \mathrm{PO}$} & 0.1 \\
\hline 577.1679 & $\mathrm{C}_{36} \mathrm{H}_{26} \mathrm{P}_{2} \mathrm{O}_{3} \mathrm{LiH}_{2}{ }^{+}$ & {$\left[\mathrm{DBFPO}+\mathrm{LiH}_{2}\right]^{+}$} & 1.7 \\
\hline 943.2475 & $\mathrm{C}_{60} \mathrm{H}_{43} \mathrm{LiO}_{5} \mathrm{P}_{3}{ }^{+}$ & $\mathrm{LiQ}+\mathrm{DBFPO}$ & -1.4 \\
\hline 1019.2789 & $\mathrm{C}_{66} \mathrm{H}_{47} \mathrm{LiO}_{5} \mathrm{P}_{3}{ }^{+}$ & $\mathrm{LiQ}+$ DBFPO + Benzene & -0.6 \\
\hline 1127.2924 & $\mathrm{C}_{72} \mathrm{H}_{52} \mathrm{P}_{4} \mathrm{O}_{5} \mathrm{Li}^{+}$ & {$\left[(\mathrm{DBFPO})_{2}-\mathrm{O}+\mathrm{Li}\right]^{+}$} & 0.9 \\
\hline
\end{tabular}

In order to reveal the origin of dominant degradation at the HBL/EML interface, we investigated the exciton distribution in the emission zone using the sensing layer method developed by Forrest et al. ${ }^{30}$ For this purpose, we fabricated a series of 5 blue OLED devices (Figure 3a) with a monolayer-thick sensing layer of red-emitting Ir dopants progressing from the EBL2/EML interface to the HBL/EML interface located at 0,100, 200, 300 and $400 \AA$ depths in the EML. The red emission intensity increased towards the HBL/EML layer (Figure 3a) indicating a large population of excitons close to the HBL. This exciton profile reveals that high energy excitons or polarons from exciton-exciton and exciton-polaron interactions generate most likely close to the HBL/EML interface, where unfavourable chemical reactions with the hole blocking layer DBFPO cause device degradation. ${ }^{11}$ The distribution of the degradation products also shows that mobile $\mathrm{Li}^{+}$ions diffused from ETL into the HBL and deeply to the EML where the exciton density was maximized. The direct driving force of $\mathrm{Li}^{+}$diffusion towards the HBL/EML interface is not clear at the moment, because it is expected that mobile $\mathrm{Li}^{+}$ions may be attracted to the $\mathrm{Al}$ cathode due to the external electric field. The laser-irradiated device $\left(\mathrm{PL}_{55}\right)$ showed the identical chemical identities for the degradation products to those in the current-aged devices $T_{95}$ and $T_{70}$. These results indicated that the interfacial region with higher exciton density or high energy excitons possibly caused the unfavourable chemical reactions and diffusion through direct bond cleavage and photothermal effects.

We also performed photoluminescence $(\mathrm{PL})$ measurements for a quantitative analysis on blue emitting dopant degradation (Figure 3b). Whereas electroluminescence (EL) decreased to $95 \%$ and $70 \%$ in $\mathrm{T}_{95}$ and $\mathrm{T}_{70}$, respectively, the $\mathrm{PL}$ intensity was $98.2 \%$ and $92.9 \%$ from the corresponding devices. This indicates that the device degradation in the aged devices $T_{95}$ and $T_{70}$ does not mainly originate from blue Ir dopants, which contributes only a $7 \%$ to the total luminescence loss of the device $T_{70}$.

A device with a slightly modified host material (mCBP-CN) presented approximately two orders of magnitude longer operational lifetime to $T_{70}$ (Figure 4a). We applied our nanoscale depth profiling method to measure the DBPFO-related degradation products and observed that these are present in much 
less abundance, which indicates that a slight change in the host material may cause a shift of exciton distribution, resulting in less accumulation of degradation products and prolonged device operational lifetime. In fact, Sim et al. ${ }^{18}$ showed that the blue OLED device containing mCBP-CN host with the identical device structure except the EML thickness has a long operational lifetime $L T_{50}$ of 431 hours at $500 \mathrm{~cd} / \mathrm{m}^{2}$ and the excitons within the EML distributed towards the HTL/EML interface. These results provide good evidence that our nanoscale chemical depth profiling method can successfully be used in the design and optimisation of new materials to achieve longer lifetimes.

\section{Discussion}

We investigated the degradation mechanism of highly energy-efficient blue phosphorescent OLEDs using GCIB-coupled OrbiSIMS as a nanoscale chemical depth probe in a layer-specific manner. We identified molecules characteristic of device degradation which are located in the ETL and EML and are identified as reaction products of DBFPO based on their layer-specific location and accurate mass measurement. The measurements have a depth resolution of better than $7 \mathrm{~nm}$ (less than $1 \mathrm{~nm}$ layer of material is consumed per high-resolution mass spectrum) with high mass resolving power, which enabled us to pinpoint the precise information on blue OLED degradation. This has previously not been attainable using any other state-of-the-art analytical techniques or traditional methods such as DESI or LC-MS. The OLED device degradation at the ETL/EML interface was confirmed by a sensing layer method that revealed that the exciton distribution in the emission zone is most intense at the same interface. A clear relationship between the intensity of the identified degradation products and device lifetime was found. Combined with device characteristics and PL measurements, we showed that the relatively short operational lifetime of the blue phosphorescent OLED with mCBP host originated from the interfacial degradation. We used the method to study a device produced with a different host material which exhibits a longer lifetime and showed that the DBPFO degradation products were observed but with much less abundance, indicating the utility of our method for guiding material optimisation to minimise degradation and optimise device lifetime.

\section{Methods}

Materials, Device fabrication, and characterization. Chemicals were purchased from commercial suppliers (Sigma-Aldrich, Wako Pure Chemical Industries, Tokyo Chemical Industry) and they were used without further purification. Synthesis of the blue Ir dopant material and fabrication details were previously described. ${ }^{6,13,18,31}$ Blue phosphorescent, bottom-emitting OLED devices were fabricated to analyse their performance and degradation mechanism using vacuum evaporating techniques with a device structure of ITO (50 nm)/ HATCN(HIL, $10 \mathrm{~nm}) / \mathrm{NPB}$ (HTL, $50 \mathrm{~nm}$ )/ TCTA (EBL, $5 \mathrm{~nm}$ )/ mCP (EBL, 5 nm)/ mCBP:Ir dopant (EML, $40 \mathrm{~nm}$ )/ DBFPO (HBL, $10 \mathrm{~nm}$ )/ DBFPO:LiQ (ETL, $30 \mathrm{~nm}$ )/ LiQ (EIL, $1 \mathrm{~nm}) / \mathrm{Al}$ (100 nm), where ITO is indium tin oxide, HATCN is 1, 4, 5, 8, 9, 11-hexaazatriphenylene-hexacarbonitrile, NPB is $N, N$-di(1-naphthyl)- $N, N$-diphenyl-(1,1'-biphenyl)-4,4'-diamine, TCTA is Tris(4-carbazoyl-9ylphenyl)amine, $\mathrm{mCP}$ is $1,3-\mathrm{Bis}(\mathrm{N}$-carbazolyl)benzene, host material mCBP is 3,3'-Di(9H-carbazol-9- 
yl)-1,1'-biphenyl, DBFPO is 2,8-bis(diphenylphosphineoxide)-dibenzofuran, and LiQ is lithium quinolinate. The organic layers of HIL, HTL, EBL, EML, HBL, and EIL served as hole injection, hole transporting, emitting, electron blocking, electron transporting, and electron injection, respectively. The organic and metal layers were deposited consecutively on pre-cleaned ITO glass substrates by using a thermal evaporation system at a pressure less than $1.0 \times 10^{-6} \mathrm{mbar}$. The deposition rates of the organic, Liq, and metal layers were $0.1,0.01$, and $1 \mathrm{~nm} \mathrm{~s}^{-1}$, respectively. The active device area was $2 \times 2 \mathrm{~mm}^{2}$ and the devices were encapsulated in a $\mathrm{N}_{2}$-filled glove box prior to all the measurements. The OLED performances were characterized by measuring the current density - voltage - luminance $(J-V-L)$ and electroluminescence spectra using a programmable source meter (Keithley 2400) and a spectrometer (Photo Research Spectrascan PR-650). The device lifetime measurements (LT95, LT70) were taken in a constant current mode. LT95 and LT70 corresponded to the operation time when the percentage luminance decreased to $95 \%$ and $70 \%$, respectively. PL55 corresponded to the $55 \%$ photoluminescence $(\mathrm{PL})$ intensity of the initial PL after a 400-nm laser irradiation.

Secondary ion mass spectrometry. SIMS depth profiles were acquired using the 3D OrbiSIMS (ION-TOF $\mathrm{GmbH}$, Muenster, Germany) which is a dual analyser SIMS instrument incorporating an Orbitrap ${ }^{\mathrm{TM}}$ mass analyser (Thermo Fisher Scientific, Bremen, Germany) and a time-of-flight (ToF) mass analyser. Secondary ions are extracted through a single set of extraction optics and then an ion-optical switch can send the secondary ions to either of the analysers. The instrument is equipped with a $30 \mathrm{kV}$ Bi Nanoprobe liquid metal ion source and a $20 \mathrm{kV}$ gas cluster ion source. This dual beam dual analyser combination can be operated in multiple modes for spectrometry, 2D imaging and 3D imaging. Depth profiles were obtained in a single beam mode using a $5 \mathrm{keV} \mathrm{Ar}_{2500}{ }^{+}$gas cluster ion beam (GCIB) as the primary ion beam and analysis of secondary ions using the Orbitrap mass analyser. The average DC primary ion beam was $100 \mathrm{pA}$. The beam was set to scan an area of $320 \mu \mathrm{m} \times 320 \mu \mathrm{m}$ of which secondary ions were collected from the central $200 \mu \mathrm{m} \times 200 \mu \mathrm{m}$ of the crater. For comparison, depth profiles were also obtained in a dual beam mode where the $5 \mathrm{keV} \mathrm{Ar}_{2500}{ }^{+}$beam was used for sputtering the sample but a 30 $\mathrm{keV} \mathrm{Bi}_{3}{ }^{+}$(average current $0.15 \mathrm{pA}$ ) was used as the analysis beam, and secondary ions were analysed using the ToF analyser (Supplementary Note 2 and Supplementary Figure 8). Electron flooding was used for charge compensation in both modes. Prior to depth profiling, the encapsulation was removed from the devices and the Al electrode layer was stripped off using sticky tape. The removal of the Al electrode is required as the GCIB does not effectively sputter metals. ${ }^{32}$

Photoluminescence spectroscopy. Photoluminescence spectra were taken using a commercial fluorescence spectrometer (Picoquant, Fluotime 300). Pristine and degraded devices with a pixel size of 2 $\mathrm{mm} \times 2 \mathrm{~mm}$ were irradiated with a 379-nm diode laser for photoexcitation. The photoluminescence signal from each device was recorded using a PMT detector through a motorized scanning monochromator. At least five independent measurements per each pixel were recorded for statistical analysis.

Liquid chromatography mass spectrometry. The liquid chromatographic separations were conducted using a high-performance liquid chromatography system equipped with a C18 column $(2.1$ x $150 \mathrm{~mm})$ 
and a photodiode array detector for UV detection (Thermofisher Ultimate 300). The HPLC system was coupled to an Orbitrap mass spectrometer (Thermofisher Velos Pro) with atmospheric pressure chemical ionization. For LC-MS sample preparations, a $2 \mathrm{~mm} \times 2 \mathrm{~mm}$ OLED pixel was cut and dissolved in a $50 \mu \mathrm{L}$ of tetrahydrofuran, followed by centrifugation to remove electrodes and glass substrate. A $30 \mu \mathrm{L}$ of the supernatant was transferred for a subsequent LC-MS analysis. The mobile phase flow rate of $\mathrm{H}_{2} \mathrm{O}$ and tetrahydrofuran gradient were $0.2 \mathrm{~mL} \mathrm{~min}^{-1}$ and the injection volume was $3 \mu \mathrm{L}$. All mass spectra were collected in positive ion mode.

\section{Declarations}

\section{Data availability}

The experimental data that supports the Figures and Tables of this study are available from the corresponding authors upon reasonable request.

\section{Supplementary information}

Experimental details and additional results of this study can be found in the supplementary information (PDF).

\section{Acknowledgements}

This work forms part of the OrbiSIMS project in the Life Science and Health programme of the National Measurement System of the UK Department of Business, Energy and Industrial strategy.

\section{Author contribution}

All authors contributed to the manuscript and approved the final manuscript. The concept was devised by SS, RH, JW, WSJ, JCL and ISG. RH acquired the SIMS data. SS, RH, GFT, JCL and ISG interpreted the data. SS and YMS performed optical characterization. LM acquired Orbitrap MS/MS data. SP, YS and SS conducted LCMS and degradation analysis. JK and HC conducted device fabrication and characterization. HJB and YJ conducted material synthesis. GFT conducted the multivariate study and identified additional PL degradation mechanism. RH, SS, GFT, LM, JHK and ISG wrote the paper. ISG, JHK and HSL oversaw the study.

\section{Competing interests}

The authors declare no competing financial interests.

\section{References}

1. Sudheendran Swayamprabha, S. et al. Approaches for Long Lifetime Organic Light Emitting Diodes. Advanced Science vol. 8 (2021). 
2. Ihn, S. G. et al. An Alternative Host Material for Long-Lifespan Blue Organic Light-Emitting Diodes Using Thermally Activated Delayed Fluorescence. Adv. Sci. 4, (2017).

3. Kang, Y. J., Han, S. H. \& Lee, J. Y. Lifetime enhancement of blue thermally activated delayed fluorescent devices by separated carrier channels using dibenzofuran-triazine type hosts. J. Ind. Eng. Chem. 62, (2018).

4. Ahn, D. H. et al. Highly efficient blue thermally activated delayed fluorescence emitters based on symmetrical and rigid oxygen-bridged boron acceptors. Nat. Photonics 13, (2019).

5. Jeon, S. O. et al. High-efficiency, long-lifetime deep-blue organic light-emitting diodes. Nat. Photonics 15, (2021).

6. Aziz, H. \& Popovic, Z. D. Degradation phenomena in small-molecule organic light-emitting devices. Chemistry of Materials vol. 16 (2004).

7. So, F. \& Kondakov, D. Degradation mechanisms in small-molecule and polymer organic light-emitting diodes. Adv. Mater. 22, (2010).

8. Scholz, S., Kondakov, D., Lüssem, B. \& Leo, K. Degradation mechanisms and reactions in organic light-emitting devices. Chemical Reviews vol. 115 (2015).

9. Aziz, H. Degradation mechanism of small molecule-based organic light-emitting devices. Science (80-.). 283, (1999).

10. Kim, S. et al. Degradation of blue-phosphorescent organic light-emitting devices involves excitoninduced generation of polaron pair within emitting layers. Nat. Commun. 9, (2018).

11. Kondakov, D. Y., Lenhart, W. C. \& Nichols, W. F. Operational degradation of organic light-emitting diodes: Mechanism and identification of chemical products. J. Appl. Phys. 101, (2007).

12. Sivasubramaniam, V. et al. Fluorine cleavage of the light blue heteroleptic triplet emitter Flrpic. J. Fluor. Chem. 130, (2009).

13. Ninomiya, S. et al. Precise and fast secondary ion mass spectrometry depth profiling of polymer materials with large Ar cluster ion beams. Rapid Commun. Mass Spectrom. 23, (2009).

14. Scholz, S., Walzer, K. \& Leo, K. Analysis of complete organic semiconductor devices by laser desorption/ionization time-of-flight mass spectrometry. Adv. Funct. Mater. 18, (2008).

15. Moraes, I. R. De, Scholz, S., Lüssem, B. \& Leo, K. Analysis of chemical degradation mechanism within sky blue phosphorescent organic light emitting diodes by laser-desorption/ionization time-of-flight mass spectrometry. Org. Electron. 12, (2011).

16. Seifert, R. et al. Chemical degradation mechanisms of highly efficient blue phosphorescent emitters used for organic light emitting diodes. Org. Electron. 14, (2013).

17. Paulson, A. E., Forsman, T. T. \& Lee, Y. J. Three-Dimensional Profiling of OLED by Laser Desorption Ionization-Mass Spectrometry Imaging. J. Am. Soc. Mass Spectrom. 31, (2020).

18. Sim, B. et al. Comprehensive model of the degradation of organic light-emitting diodes and application for efficient, stable blue phosphorescent devices with reduced influence of polarons. Phys. Rev. Appl. 14, (2020). 
19. Ninomiya, S. et al. Analysis of organic semiconductor multilayers with Ar cluster secondary ion mass spectrometry. in Surface and Interface Analysis vol. 43 (2011).

20. Shard, A. G. et al. Argon cluster ion beams for organic depth profiling: Results from a VAMAS interlaboratory study. Anal. Chem. 84, 7865-7873 (2012).

21. Aizawa, N. et al. Solution-processed multilayer small-molecule light-emitting devices with highefficiency white-light emission. Nat. Commun. 5, (2014).

22. Bell, B. M. et al. Degradation of Hole Transport Materials via Exciton-Driven Cyclization. ACS Appl. Mater. Interfaces 9, (2017).

23. Vasilopoulou, M. et al. High efficiency blue organic light-emitting diodes with below-bandgap electroluminescence. Nat. Commun. 12, (2021).

24. Noël, C. et al. Depth Profiling of Organic Light-Emitting Diodes by ToF-SIMS Coupled with WaveletPrincipal Component Analysis. ACS Appl. Polym. Mater. 1, (2019).

25. Passarelli, M. K. et al. The 3D OrbiSIMS-label-free metabolic imaging with subcellular lateral resolution and high mass-resolving power. Nat. Methods nmeth.4504 (2017) doi:10.1038/nmeth.4504.

26. lida, S. ichi et al. Time-of-flight secondary ion tandem mass spectrometry depth profiling of organic light-emitting diode devices for elucidating the degradation process. Rapid Commun. Mass Spectrom. 34, (2020).

27. Giebink, N. C. et al. Intrinsic luminance loss in phosphorescent small-molecule organic light emitting devices due to bimolecular annihilation reactions. J. Appl. Phys. 103, (2008).

28. Trindade, G. F., Abel, M. L. \& Watts, J. F. simsMVA: A tool for multivariate analysis of ToF-SIMS datasets. Chemom. Intell. Lab. Syst. 182, 180-187 (2018).

29. Shim, S., Choi, E., Kim, H. \& Yun, J. Y. Degradation Behaviors of NPB Molecules upon Prolonged Exposure to Various Thermal Stresses under High Vacuum below 10-4Pa. ACS Omega 5, (2020).

30. Zhang, Y., Lee, J. \& Forrest, S. R. Tenfold increase in the lifetime of blue phosphorescent organic lightemitting diodes. Nat. Commun. 5, (2014).

31. Metz, S. et al. Metal complexes comprising azabenzimidazole carbene ligands and the use thereof in OLEDs. U.S. Pat. Appl. Publ. 73pp. (2012).

32. Seah, M. P. Universal equation for argon gas cluster sputtering yields. J. Phys. Chem. C 117, 1262212632 (2013).

\section{Figures}

\section{Figure 1}


High-resolution chemical depth profiling of OLED devices. a Experimental schematics of the OrbiSIMS instrument and a OLED device. See Methods for abbreviations. b Depth resolution estimation using a reference 1-nm-thick delta layer of blue Ir dopant in a host matrix. $\mathbf{c}$ Depth profiles for 8 parent ions characteristic of the device architecture displayed above to aid interpretation. Further details of mass accuracy and mass resolving power are given in Supplementary Table 2. $\mathbf{d}$ High-resolution mass spectra from each region in the depth profile.

\section{Figure 2}

High resolution MS is used to identify five degradation ions from their mass and location in the device architecture. a Structure and assignment for degradation products in the blue OLED device. b Depth profiles for the $T_{100}$ (pristine), $T_{95}$ and $T_{70}$ and $\mathrm{PL}_{55}$ devices respectively from bottom to top. Degradation product ions are putatively assigned as shown in Table 1. The host dopants PBH01 $(\mathrm{m} / z$ 484.1934, $\left.\mathrm{C}_{36} \mathrm{H}_{24} \mathrm{~N}_{2}{ }^{+}\right)$and PBD01 $\left(\mathrm{m} / z\right.$ 1006.2596, $\left.\mathrm{C}_{51} \mathrm{H}_{33} \mathrm{~N} 121 \mathrm{r}^{+}\right)$are shown in grey and black respectively to aid orientation in the device architecture. c Relative intensities of peaks related to potential degradation products calculated as the normalised intensity in each of the devices relative to the normalised intensity in the pristine. Normalisation is done by dividing peak intensities by total ion counts of a depth profile.

\section{Figure 3}

\section{Exciton distribution in the emission zone and photoluminescence of blue OLED devices. a}

Electroluminescence measurements from 5 test devices with monolayer-thick sensing layers of red lightemitting Ir dopants at 0,100,200,300, $400 \AA$ from the EBL/EML interface. EL intensity normalized to the $400 \AA ̊$ device measurement. b Photoluminescence measurements of blue dopants for the $T_{100}, T_{95}, T_{70}$ and $\mathrm{P}_{59}$ devices.

\section{Figure 4}

Comparison of operational device lifetimes and interfacial degradation products. a Luminance and EQE change as a function of time for an initial $1000 \mathrm{~cd} / \mathrm{m}^{2}$ for blue OLED devices with two host materials (mCBP and mCBP-CN) b Relative intensity of DBFPO:Liq degradation product (1127.2924 u) in the two blue devices at different stages of degradation.

\section{Supplementary Files}

This is a list of supplementary files associated with this preprint. Click to download. 
- BlueOLEDdegradationSIv03.docx 